\title{
Successful Intravenous Thrombolysis for Acute Stroke Caused by Polycythemia Vera
}

\author{
Ashkan Mowla, ${ }^{1,}{ }^{*}$ Harshit Shah, ${ }^{1}$ Navdeep S. Lail, ${ }^{1}$ and Peyman Shirani ${ }^{1}$ \\ ${ }^{1}$ Stroke Division, Department of Neurology, University at Buffalo, State University of New York, 100 High Street, Buffalo, NY, 14203 \\ "Corresponding author: Ashkan Mowla, Department of Neurology, Gates Vascular Institute, School of Medicine and Biomedical Sciences, State University of New York at \\ Buffalo, 100 High Street, Buffalo, NY 14203. Tel: +01-7168597540, Fax: +01-7168592430, E-mail: mowla_a@yahoo.com
}

Received 2017 April 10; Revised 2017 September 25; Accepted 2017 October 08.

Keywords: Stroke, Thrombolysis, Polycythemia, Vera

\section{Dear Editor,}

Polycythemia vera (PCV) is a chronic clonal myeloproliferative disorder characterized by increased red-cell mass; elevated white-cell and platelet counts are also common (1). Thrombotic events are the presenting symptoms in $20 \%$ of patients with PCV and the major cause of morbidity and mortality in untreated patients. Thrombosis in PCV is attributed to several factors including hyperviscosity of the blood causing cerebral blood flow disturbance and platelet marginalization with increased contact to vessel and subsequent platelet activation. Approximately 70 $\%$ of these thrombotic events are cerebrovascular $(1,2)$. Intravenous recombinant tissue plasminogen activator (IV rtPA) is the only FDA-approved pharmacological therapy for acute ischemic stroke (AIS) (3). To the best of our knowledge, there is no report in the literature on intravenous thrombolysis (IVT) for AIS caused by PCV.

We present the case of a 55 year-old gentleman with known diagnosis of PCV and no other significant medical history who presented to the emergency department (ED) with acute onset left facial droop , slurred speech and left arm weakness and was treated with IV rtPA (0.9 mg/kg) within 3 hours of symptom onset. His national institutes of health stroke scale (NIHSS) was 6 on admission. His initial evaluation in the ED included a EKG which showed normal sinus rhythm with no ischemic change, a non-contrast head CT which was unremarkable for any acute abnormality, CT angiogram of head and neck which did not show any significant vascular stenosis or intracranial proximal large vessel occlusion, blood glucose of $108 \mathrm{mg} / \mathrm{dL}$ and blood pressure of $138 / 85 \mathrm{mmHg}$. A complete blood count was obtained which showed WBC: 28,100 cells/mcL, Hemoglobin: $14.4 \mathrm{~g} / \mathrm{dL}$, hematocrit: $49.4 \%$ and Platelet count: 1,794,000 cells/mcL. Post IVT, he was admitted to neuro intensive care unit with frequent neuro and vital sign checks for 24 hours. Head CT was repeated in 24 hour post IVT which showed no intracranial hemorrhage. Stroke work up was performed to evaluate for any potential cause for AIS besides PCV. Transthoracic echocardiogram and cardiac magnetic resonance imaging (MRI) with contrast (4) did not show any cardiac source for emboli. 48 hours cardiac monitoring during the hospital course did not show any paroxysmal atrial fibrillation or flutter. Given no other potential cause for AIS was discovered during our extensive work up, it is reasonable to consider PCV as the mechanism for AIS in our patient. Brain MRI Diffusion weighted image done on the 2nd day of admission shows acute infarct in the right MCA territory (Figure 1). Patient was discharged on day 3 after admission with NIHSS of 0 and intact neurological exam on aspirin $81 \mathrm{mg}$ p.o daily.

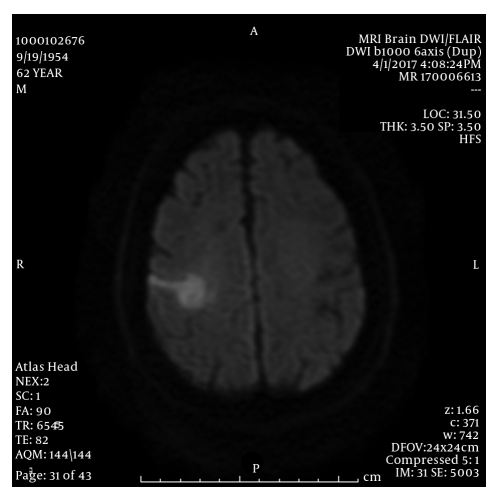

Figure 1. Brain MRI Diffusion Weighted Image Shows Diffusion Restriction in the Right MCA Territory Consistent with Acute to Subacute Infarct

In conclusion, IV rtPA for AIS caused by PCV might be safe and effective and needs to be considered in such pa- 
tients. To the best of our knowledge, we report herein the first case in the literature describing the use of IVT for AIS caused by PCV.

\section{Footnotes}

Financial Disclosure: Authors have no relevant financial disclosure to report.

Funding/Support: None.

\section{References}

1. Vannucchi AM, Kiladjian JJ, Griesshammer M, Masszi T, Durrant S, Passamonti F, et al. Ruxolitinib versus standard therapy for the treatment of polycythemia vera. N Engl J Med. 2015;372(5):426-35. doi: 10.1056/NEJMoa1409002. [PubMed: 25629741].

2. Kwaan HC, Wang J. Hyperviscosity in polycythemia vera and other red cell abnormalities. Semin Thromb Hemost. 2003;29(5):451-8. doi: 10.1055/s-2003-44552. [PubMed: 14631544].

3. Mowla A, Singh K, Mehla S, Ahmed MK, Shirani P, Kamal H. Is acute reperfusion therapy safe in acute ischemic stroke patients who harbor unruptured intracranial aneurysm? Inter J Strok. 2015;10(100):113-8.

4. Baher A, Mowla A, Kodali S, Polsani VR, Nabi F, Nagueh SF, et al. Cardiac MRI improves identification of etiology of acute ischemic stroke. Cerebrovasc Dis. 2014;37(4):277-84. doi: 10.1159/000360073. [PubMed: 24819735]. 